\title{
A CRITERION FOR ABSOLUTE CESÀRO SUMMA- BILITY OF NEGATIVE ORDER OF A FOURIER SERIES
}

\section{by YUNG-MING CHEN}

(Received 25th May 1964; revised MS. received 31st December 1964)

1. Let $f(x)$ be integrable $L(0,2 \pi)$ and periodic with period $2 \pi$, and let $\psi(t)$ be the conjugate function of $\frac{1}{2}\{f(x+t)+f(x-t)\}$ with respect to the variable $t$, where $x$ is considered as an arbitrary constant. The following theorems are due to K. K. Chen (1), (2), pp. 111-124.

Theorem A. Suppose that $p>1,0<k<1, p k>1$; and that at the point $x$,

$$
\int_{0}^{\pi}|\psi(t+h)-\psi(t-h)|^{p} d t=O\left(h^{p k}\right),
$$

as $h \rightarrow+0$. Then the Fourier series of $f(t)$ at the point $t=x$ is summable $|C, \alpha|$, for $\alpha>\alpha_{0}=\max \left(\frac{1}{2}-k, \frac{1}{p}-k\right)$.

Theorem B. Suppose that at the point $x$, there is a number $q \geqq 0$ such that $q+p k>1$ and that as $h \rightarrow+0$,

$$
\int_{0}^{\pi}|\psi(t+h)-\psi(t-h)|^{p} t^{-q} d t=O\left(h^{p k}\right) .
$$

Then the Fourier series of $f(t)$ at $t=x$ is summable $|C, \alpha|$, when

$$
\alpha>\alpha_{0}=\max \left(\frac{1}{2}-k, \frac{1}{p}-k\right),
$$

and is summable $(C, \beta)$ for $\beta>-k$.

Theorem C. If $F(z)=\Sigma c_{n} z^{n}\left(z=r e^{i \theta}\right)$ is regular in the unit circle $|z|<1, \uparrow$ and if for a positive integer $j$,

$$
\int_{-\pi}^{\pi} \frac{\left|F^{(j)}(z)\right|^{p}}{|1-z|^{q}} d \theta=O\left\{(1-r)^{p(k-j)}\right\} \quad\left(z=r e^{i \theta}\right),
$$

$\dagger$ In (2), p. 112, Theorem 4, it is assumed that $F(z)$ is also regular at $z=1$, but it seems likely that this is unnecessary. I remark also that in the proof of Theorem 3 in (2), p. 112, Theorem 4, in p. 112 is used; but the condition for regularity at $z=1$ is not provided there. 
as $r \rightarrow 1$, where $F^{(j)}(z)$ is the jth derivative of $F(z)$, then the series $\Sigma c_{n}$ is summable $|C, \alpha|$, whenever $\alpha>\alpha_{0}=\max \left(\frac{1}{2}-k, \frac{1}{p}-k\right)$, and $0 \leqq q<1, p>1$, $0<k<1, p k+q>1$.

Theorems A, B, C include a number of criteria for absolute convergence and absolute Cesàro summability of Fourier series due to Hardy and Littlewood (8), Hyslop (10), Chow (3), (4). In this paper, we shall consider further extensions of Theorems A, B, C for absolute Cesàro summability " to index $\mu$ " in the sense of T. M. Flett (5), (6), (7). The series $\Sigma a_{n}$ is said to be summable $|C, \alpha|_{\mu}$, where $\mu \geqq 1, \alpha>-1$, if the series $\Sigma n^{\mu-1}\left|\sigma_{n}^{\alpha}-\sigma_{n-1}^{\alpha}\right|^{\mu}$ is convergent. For any given series $\sum_{0}^{\infty} a_{n}$, we write $\sigma_{n}^{0}=S_{n}^{0}=a_{0}+a_{1}+\ldots+a_{n}$,

$S_{n}^{\alpha}=\sum_{\nu=0}^{n} A_{v}^{\alpha-1} S_{v}^{0}, \tau_{n}^{0}=T_{n}^{0}=n a_{n}, T_{n}^{\alpha}=\sum_{v=0}^{n} A_{n-v}^{\alpha-1} T_{v}^{0}, \quad \sigma_{n}^{\alpha}=S_{n}^{\alpha} / A_{n}^{\alpha}, \tau_{n}^{\alpha}=T_{n}^{\alpha} / A_{n}^{\alpha}$ where

$$
A_{n}^{\alpha}=\left(\begin{array}{c}
\alpha+n \\
n
\end{array}\right)=\frac{(\alpha+1) \ldots(\alpha+n)}{n !}=\frac{\Gamma(n+\alpha+1)}{\Gamma(n+1) \Gamma(\alpha+1)},
$$

$n>1, \alpha>-1, A_{0}^{\alpha}=1$. Then we also have $\dagger$

$$
\begin{aligned}
\tau_{n}^{\alpha} & =n\left(\sigma_{n}^{\alpha}-\sigma_{n-1}^{\alpha}\right), \\
\tau_{n}^{\alpha} & =-\alpha\left(\sigma_{n}^{\alpha}-\sigma_{n}^{\alpha-1}\right) \quad(\alpha>0) .
\end{aligned}
$$

In view of (1.4), we may restate the definition of summation $|C, \alpha|_{\mu}$ in terms of the series $\sum_{1}^{\infty} n^{-1}\left|\tau_{n}^{\alpha}\right|^{\mu}$. Thus the series $\Sigma a_{n}$ is summable $|C, \alpha|_{\mu}$ if and only if the series $\Sigma n^{-1}\left|\tau_{n}^{\alpha}\right|^{\mu}$ is convergent. . T. M. Flett further considered an extension of $|C, \alpha|_{\mu}$ summability: The series $\Sigma a_{n}$ is said to be summable $|C, \alpha, \gamma|_{\mu}$, where $\mu \geqq 1, \alpha>-1$, if the series $\Sigma n^{\mu \gamma+\mu-1}\left|\sigma_{n}^{\alpha}-\sigma_{n-1}^{\alpha}\right|$ is convergent. Thus summability $|C, \alpha|_{\mu}$ is the special case of summability $|C, \alpha, \gamma|_{\mu}$ when $\gamma=0$.

Theorem 1. $\ddagger$ Let $F(z)=\sum_{n=0}^{\infty} c_{n} z^{n}$ be regular in $|z|<1$. If the following conditions are satisfied:

(i) $p>1$,

(ii) $0<k \leqq 1$,

(iii) $0 \leqq q \leqq 1-k$,

(iv) $0 \leqq y<\min \left(\frac{1}{2},(q-1) / p+k\right)$,

$\dagger$ See (11) and (5), p. 114.

$\ddagger I$ am indebted to the referee for pointing out a mistake and for valuable suggestions which brought material improvements of the conditions of this theorem. 
(v) $\alpha>\alpha_{0}=\max \left(\frac{1}{2}-k+\gamma, \frac{1}{p}-k+\gamma\right)$

(vi) $1 \leqq \mu \leqq \frac{\min (p, 2)}{\min (p, 2)-1}$,

(vii) for some positive integer $j$,

$$
\int_{-\pi}^{\pi} \frac{\left|F^{(j)}(z)\right|^{p}}{|1-z|^{q}} d \theta=O\left\{(1-r)^{p(k-j)}\right\}
$$

as $r \rightarrow 1$, where $z=r e^{i \theta}$, then the series $\Sigma c_{n}$ is summable $|C, \alpha, \gamma|_{\mu}$.

Theorem 2. Let the conditions (i)-(vi) of Theorem 1 be satisfied and let

$$
\int_{0}^{\pi}|\psi(t+h)-\psi(t-h)|^{p} t^{-q} d t=O\left(h^{p h}\right)
$$

as $h \rightarrow+0$, then the Fourier series of $f(t)$ is summable $|C, \alpha, \gamma|_{\mu}$ at $t=x$.

Corollary. If $p>1,0<1 / p<k \leqq 1$, and $\alpha>\alpha_{0}=\max \left(1 / p-k, \frac{1}{2}-k\right)$, $1 \leqq \mu \leqq \frac{\min (p, 2)}{\min (p, 2)-1}$, and if

$$
\int_{0}^{\pi}|\psi(t+h)-\psi(t-h)|^{p} d t=O\left(h^{p k}\right)
$$

as $h \rightarrow+0$, then the Fourier series of $f(t)$ is summable $|C, \alpha|_{\mu}$ at $t=x$.

Theorem 3. Suppose that $f(t) \sim \sum_{n=0}^{\infty} A_{n}(t)$ and that the following inequalities are satisfied:

(i) $1 \leqq p_{1} \leqq 2 \leqq p_{2}$,

(ii) $k_{j}>0(j=1,2)$,

(iii) $0<\gamma<k^{\prime}-\frac{1}{2}<k^{\prime}=\left\{p_{1} k_{1}\left(p_{2}-2\right)+p_{2} k_{2}\left(2-p_{1}\right)\right\} / 2\left(p_{2}-p_{1}\right) \leqq 1$,

(iv) $\alpha>\frac{1}{2}-k^{\prime}+\gamma$,

(v) $1 \leqq \mu \leqq 2$.

Furthermore, if

$$
\int_{-\pi}^{\pi}|\psi(t+h)-\psi(t-h)|^{p_{j}} d t=O\left(h^{p j k}\right) \quad(j=1,2),
$$

as $h \rightarrow+0$, then the Fourier series of $f(t)$ is summable $|C, \alpha, \gamma|_{\mu}$ at $t=x$. In particular, if the function $\psi$ in (1.9) is replaced by $f$, if $1 \leqq \min \left(p_{1} k_{1}, p_{2} k_{2}\right)$ $<\max \left(p_{1} k_{1}, p_{2} k_{2}\right)$, and if (iii) is replaced by $k^{\prime} \leqq 1$, then the Fourier series of $f(t)$ is summable $|C, \alpha|$ at $t=x$. 
2. For the proofs of Theorems 1, 2, 3, we require the following lemmas:

Lemma 1. Suppose that $q \geqq 0$, that the odd function $\psi(\theta)$ is the imaginary part of the boundary function

$$
g(\theta)=F\left(e^{i \theta}\right)=\phi(\theta)+i \psi(\theta),
$$

where $F(z)$ is regular in the unit circle $|z|<1$. Then the condition (1.7) with $p>1,0<k \leqq 1$ implies

$$
\int_{-\pi}^{\pi}\left|F^{(j)}\left(r e^{i \theta}\right)\right|^{p}|\theta|^{-q} d \theta=O\left\{(1-r)^{p(k-j)}\right\},
$$

for every $j=1,2, \ldots$, as $r \rightarrow 1$, where $F^{(j)}(z)$ is the jth derivative of $F(z)$.

Lemma 2. If $p>1,0<k \leqq 1, q \geqq 0, F(z)=\sum_{n=0}^{\infty} c_{n} z^{n}$ is regular in $|z|<1$, and if the boundary function (2.1) satisfies the following condition:

$$
\int_{-\pi}^{\pi}|g(\theta+t)-g(\theta-t)|^{p}|\theta|^{-q} d \theta=O\left(|t|^{p k}\right),
$$

as $t \rightarrow 0$, then the jth derivative $F^{(j)}(z)$ of $F(z)$ satisfies the relation (2.2) for every $j=1,2, \ldots$.

Lemma 3. If $p>1,0<k \leqq 1, z=r e^{i \theta}$, if $F(z)=\sum_{n=0}^{\infty} c_{n} z^{n}$ is regular in $|z|<1$ and if for some positive integer $j$,

$$
\int_{-\pi}^{\pi}\left|F^{(j)}(z)\right|^{p} d \theta=O\left\{(1-r)^{p(k-j)}\right\}
$$

as $r \rightarrow 1$, then the boundary function $g(\theta)=F\left(e^{i \theta}\right)$ satisfies the relation:

as $t \rightarrow 0$.

$$
\int_{-\pi}^{\pi}|g(\theta+t)-g(\theta-t)|^{p} d \theta=O\left(|t|^{p k}\right)
$$

Lemma 4. If the functions $F(z)=\sum_{n=0}^{\infty} c_{n} z^{n}=\sum_{n=0}^{\infty} c_{n} r^{n} e^{n i \theta}$ is regular in $|z|<1$, and if for some positive integer $j$,

$$
\int_{-\pi}^{\pi} \frac{\left|F^{(j)}(z)\right|^{p}}{|1-z|^{q}} d \theta=O\left\{(1-r)^{p(k-j)}\right\}
$$

as $r \rightarrow 1$, then (2.6) is satisfied for every $j=1,2, \ldots$, where $r>1,0<k \leqq 1$, $0 \leqq q \leqq 1-k$.

The proofs of Lemmas 1-4 are essentially due to K. K. Chen $\dagger(1)$, (2), pp. 116-118, except the case when $k=1$. As a matter of fact, the proofs of Lemmas 1, 2, 4 have been given in (2) and when $k=1$ the results are still

$\dagger$ Here it should be remarked that in Lemma 1, the condition (1.7) with $q \geqq 0$ implies $\psi(t) \in L^{p}(-\pi, \pi)$. This result is due to Hardy and Littlewood (9), p. 566 . 
valid. So we omit the proofs here. But for the proof of Lemma 3 which is Lemma 4 in (2), p. 117, K. K. Chen's method is to reduce the case for general $j$ by $j-1$, and then the result follows from mathematical induction. In fact, the special case when $j=1, k=1$ is still true. $\dagger$ So the result for the general case $0<k \leqq 1$ follows at once.

Lemma 5. $\ddagger$ (a) Let $r \geqq k>1, \gamma \geqq 0, \alpha>\gamma-1, \beta \geqq \alpha+\frac{1}{k}-\frac{1}{r}$. Then if $\sum_{0}^{\infty} a_{n}$ is summable $|C, \alpha, \gamma|_{k}$, it is summable $|C, \beta, \gamma|_{r}$ and

$$
\left\{\Sigma n^{r \gamma-1}\left|\tau_{n}^{\beta}\right|^{r}\right\}^{1 / r} \leqq K\left\{\Sigma n^{k \gamma-1}\left|\tau_{n}^{\alpha}\right|^{k}\right\}^{1 / k} .
$$

(b) Let $\mu \geqq 1, \gamma \geqq 0, \alpha>\gamma-1, \gamma>v$, where $v$ is not necessarily non-negative, $\beta \geqq \alpha-\gamma+v, \beta>-1$. Then if $\sum_{0}^{\infty} a_{n}$ is summable $|C, \alpha, \gamma|_{\mu}$, it is summable $|C, \beta, v|_{\mu}$ and

$$
\Sigma n^{k v-1}\left|\tau_{n}^{\beta}\right|^{\mu} \leqq K \Sigma n^{\mu \gamma-1}\left|\tau_{n}^{\alpha}\right|^{\mu} .
$$

3. We come now to the proof of Theorem 1 for $\mu>1$. Suppose that the conditions (i)-(vii) are satisfied. Then by (ii), (iii), (vii) and Lemma 4 , the relation (1.6) holds for all $j$.

Consider first the case $1<p \leqq 2$. In view of Lemma $5(a)$, it is sufficient to prove that when $\alpha_{0}<\alpha \leqq q \mid p$,

$$
\sum_{v=1}^{n} v^{\mu \gamma-1}\left|\tau_{v}^{\alpha}\right|^{\mu}=O(1)
$$

as $n \rightarrow \infty$, provided that the following conditions are satisfied:

$$
\alpha>-1, \alpha<1-k, \alpha \leqq q / p, \alpha>1 / p-k+\gamma, 1<\mu \leqq p^{\prime} .
$$

In fact, by virtue of (iii), we have $q / p<q \leqq 1-k$, and since, by (iv) $q / p>1 / p-k+\gamma$, the conditions (3.2) are satisfied when $\alpha_{0}<\alpha \leqq q / p$. If this can be established, then by virtue of Lemma 5 (a), (3.1) is satisfied for all $\alpha>\alpha_{0}$ (since $\alpha_{0}>\gamma-1$ ). We have

$$
\begin{gathered}
A_{n}^{\alpha}=\left(\begin{array}{c}
n+\alpha \\
n
\end{array}\right)=\frac{(\alpha+1) \ldots(\alpha+n)}{n !}=\frac{\Gamma(n+\alpha+1)}{\Gamma(n+1) \Gamma(\alpha+1)}, \\
\sum_{n=0}^{\infty} A_{n}^{\alpha} \tau_{n}^{\alpha} z^{n}=z F^{\prime}(z)(1-z)^{-\alpha} . \S
\end{gathered}
$$

It follows from the relation

$$
B(x, y)=\int_{0}^{1} \lambda^{x-1}(1-\lambda)^{y-1} d \lambda=\frac{\Gamma(x) \Gamma(y)}{\Gamma(x+y)}
$$

$\dagger$ Cf. (8), Theorem 3.

$\ddagger$ See Flett (7), Theorem 1 and Theorem 3 .

$\S$ See Flett (5), p. 114, formula (2.1.6). 
that

$$
\begin{aligned}
\sum_{n=0}^{\infty} \tau_{n}^{\alpha} z^{n+\alpha} & =\int_{0}^{z}(z-t)^{\alpha} \frac{d}{d t}\left\{t F^{\prime}(t)(1-t)^{-\alpha}\right\} d t \\
& =\int_{0}^{z}(z-t)^{\alpha}(1-t)^{-\alpha} F^{\prime}(t) d t+\int_{0}^{z}(z-t)^{\alpha} t(1-t)^{-\alpha} F^{\prime \prime}(t) d t \\
& +\int_{0}^{z}(z-t)^{\alpha} \alpha t(1-t)^{-\alpha-1} F^{\prime}(t) d t=I_{1}+I_{2}+I_{3}, \text { say. }
\end{aligned}
$$

From Lemma 4 together with Minkowski's inequality and the relation $\alpha \leqq q / p$, we obtain

$$
\begin{aligned}
& \left\{\int_{-\pi}^{\pi}\left|I_{1}\right|^{p} d \theta\right\}^{1 / p}=\left\{\int_{-\pi}^{\pi} d \theta\left|\int_{0}^{z}(z-t)^{\alpha}(1-t)^{-\alpha} F^{\prime}(t) d t\right|^{p}\right\}^{1 / p} \\
& \quad \leqq \int_{0}^{r}(r-\rho)^{\alpha}\left\{\int_{-\pi}^{\pi} \frac{\left|F^{\prime}(t)\right|^{p}}{|1-t|^{q}} d \theta\right\}^{1 / p} d \rho=O\left\{\int_{0}^{r}(r-\rho)^{\alpha}(1-\rho)^{k-1} d \rho\right\}
\end{aligned}
$$

as $r \rightarrow 1$, where $t=\rho e^{i \theta}, z=r e^{i \theta}$. Since $\alpha>-1$, integration_by parts gives

$$
\begin{aligned}
\int_{0}^{r}(r-\rho)^{\alpha}(1-\rho)^{k-1} d \rho=\frac{r^{\alpha+1}}{1+\alpha}+ & \frac{1-k}{1+\alpha} \int_{0}^{r}(r-\rho)^{\alpha+1}(1-\rho)^{k-2} d \rho \\
& \leqq \frac{1}{1+\alpha}+\frac{1-k}{1+\alpha} \int_{0}^{r}(1-\rho)^{\alpha+k-1} d \rho .
\end{aligned}
$$

In view of (3.2), we see that $\alpha+k-1<0$. It follows from (3.4) and (3.5) that

$$
\left\{\int_{-\pi}^{\pi}\left|I_{1}\right|^{p} d \theta\right\}^{1 / p}=O\left\{(1-r)^{\alpha+k-1}\right\}
$$

as $r \rightarrow 1$. In a similar way, it can be readily shown that

$$
\left\{\int_{-\pi}^{\pi}\left|I_{2}\right|^{p} d \theta\right\}^{1 / p}=O\left\{(1-r)^{\alpha+k-1}\right\}
$$

as $r \rightarrow 1$. Since $\alpha \leqq q / p$,

$$
(1-t)^{-\alpha-1}=O\left\{(1-\rho)^{-1}|1-t|^{-q / p}\right\},
$$

it follows in a similar way that

$$
\left\{\int_{-\pi}^{\pi}\left|I_{3}\right|^{p} d \theta\right\}^{1 / p}=O\left\{\int_{0}^{r}(r-\rho)^{\alpha}(1-\rho)^{-1}(1-\rho)^{k-1} d \rho\right\}=O\left\{(1-r)^{\alpha+k-1}\right\}
$$

Hence

$$
M(p)=\left\{\sum_{v=1}^{\infty}\left|\tau_{v}^{\alpha} z^{v}\right|^{p} d \theta\right\}^{1 / p}=O\left\{(1-r)^{\alpha+k-1}\right\}
$$


as $r \rightarrow 1$. The expression $M(p)$ is a non-decreasing function of $p$. This gives

$$
M(\min (p, 2))=O\left\{(1-r)^{\alpha+k-1}\right\} .
$$

Write $P=\frac{\min (p, 2)}{\min (p, 2)-1}$, then by Hausdorff's theorem, we have

$$
\left\{\sum_{1}^{\infty}\left|\tau_{n}^{\alpha} r^{n}\right|^{P}\right\}=O\left\{(1-r)^{P(\alpha+k-1)}\right\} .
$$

Setting $r=1-1 / n$, we obtain

$$
\left\{\sum_{v=1}^{n}\left|\tau_{v}^{\alpha}\right|^{P}\right\}^{1 / P}=O\left(n^{1-\alpha+k}\right)=O\left(n^{s}\right)
$$

where $s=1-\alpha-k$. By Hölder's inequality,

$$
\sum_{v=1}^{n}\left|\tau_{v}^{\alpha}\right|^{\mu} \leqq\left\{\sum_{v=1}^{n}\left|\tau_{v}^{\alpha}\right|^{\mathrm{P}}\right\}^{\mu / \mathrm{P}} n^{(\mathrm{P}-\mu) / \mathrm{P}}=O\left\{n^{[s \mu+(p-\mu p+\mu) / p]}\right\},
$$

provided that $1 \leqq \mu \leqq P=p /(p-1)=p^{\prime}$, which is satisfied by (3.2). Then by Abel's transformation, we obtain

$$
\begin{aligned}
\sum_{\nu=1}^{n} v^{\mu \gamma-1}\left|\tau_{v}^{\alpha}\right|^{\mu} & =O\left\{\sum_{v=1}^{n-1} v^{s \mu+(p-\mu p+\mu) / p+\mu \gamma-2}+n^{\mu \gamma-1+s \mu+(p-\mu p+\mu) / p}\right\} \\
& =O(1),
\end{aligned}
$$

as $n \rightarrow \infty$, provided that $s \mu-(p+\mu p-\mu) / p<-1-\mu \gamma$, or $s<1-1 / p-\gamma$, which is equivalent to the condition $\alpha>-k+1 / p+\gamma$ in (3.2). Hence (3.1) is satisfied for $1<p \leqq 2$. Consider next the case $p>2$. Then by Hölder's inequality, we have for all $j$

$$
\begin{aligned}
\left\{\int_{-\pi}^{\pi} \frac{\left|F^{(j)}(z)\right|^{2}}{|1-z|^{w}} d \theta\right\}^{\frac{1}{2}} & \leqq\left\{\int_{-\pi}^{\pi}|1-z|^{(2 q-p w) /(p-2)} d \theta\right\}^{(p-2) / 2 p}\left\{\int_{-\pi}^{\pi} \frac{\left|F^{(j)}(z)\right|^{p}}{|1-z|^{w}} d \theta\right\}^{1 / p} \\
& =O\left\{(1-r)^{k-j}\right\},
\end{aligned}
$$

provided that $(p w-2 q) /(p-2)<1$, i.e. provided that $w<1+2(q-1) / p$. Thus provided that $w<1+2(q-1) / p$, the condition (1.6) holds for all $j$ with $p$ and $q$ replaced by 2 and $w$ respectively. Hence, we have (3.1) provided that

$$
\alpha>-1, \alpha<1-k, \alpha \leqq w / 2, \alpha>\frac{1}{2}-k+\gamma, 1<\mu \leqq 2 .
$$

In order that the statement (3.1) should not be vacuous, we require

and

$$
1-k>\frac{1}{2}-k+\gamma \text {, i.e. } \gamma<\frac{1}{2}
$$

$$
w / 2>\frac{1}{2}-k+\gamma \text {. }
$$

Take $w=1+2(q-1) / p-2 \varepsilon$, where $\varepsilon$ is chosen so that $0<\varepsilon<(q-1) / p+k-\gamma$ (this is possible by (iv)). Then (3.8) is satisfied, and $w<1+2(q-1) / p$, and hence (3.1) holds for $\alpha_{0}<\alpha<\min (1-k, w / 2)$, and therefore by Lemma 5 (a), for all $\alpha>\alpha_{0}$. This completes the proof for $\mu>1$. 
We now consider the proof of Theorem 1 for the case $\mu=1$. Let us insert $\gamma^{\prime}>\gamma$ so that for given $\alpha$, the conditions (iv), (v) of Theorem 1 can be written as:

(iv) $0 \leqq \gamma<\gamma^{\prime}<\min \left(\frac{1}{2},(q-1) / p+k\right)$,

(v) $\alpha>\alpha_{0}+\left(\gamma^{\prime}-\gamma\right)>\alpha_{0}=\max \left(\frac{1}{2}-k+\gamma, 1 / p-k+\gamma\right)$.

Then as in the proof for $\mu>1, \alpha_{0}<\alpha \leqq p / q$,

$$
\sum_{\nu=1}^{n} \nu^{\mu \gamma^{\prime}-1}\left|\tau_{v}^{\alpha}\right|^{\mu}=O(1)
$$

as $n \rightarrow \infty$, and therfore by virtue of Lemma $5(\mathrm{~b})$, we see that

$$
\sum_{\nu=1}^{n} v^{\mu \gamma-1}\left|\tau_{v}^{\alpha}\right|^{\mu}=O(1)
$$

as $n \rightarrow \infty$, for all $\alpha>\alpha_{0}$. By a similar argument as in the proof for the case $\mu>1$, we see that when $p>2$, the above relation still holds when $\alpha>\alpha_{0}$. Thus the proof of Theorem 1 is completed.

Proof of Theorem 2. Let $f(t) \sim \sum_{n=0}^{\infty} A_{n}(t)$ and write $z=r e^{i t}$,

$$
F(z)=\sum_{n=0}^{\infty} A_{n}(x) z^{n}
$$

The odd function $\psi(t)$ is the imaginary part of the boundary function of $F(z)$. By (1.7) and Lemma 1, we obtain (1.6) $\dagger$ and the result follows immediately from Theorem 1 .

Finally for the proof of Theorem 3, we see that in virtue of Hölder's inequality.

$$
\begin{aligned}
& \int_{0}^{\pi}|\psi(t+h)-\psi(t-h)|^{2} d t=\frac{1}{2} \int_{-\pi}^{\pi}|\psi(t+h)-\psi(t-h)|^{2} d t \leqq \frac{1}{2}\left\{\int_{-\pi}^{\pi}|\psi(t+h)-\psi(t-h)|^{p_{1}} d t\right\}^{\left(p_{2}-2\right) /\left(p_{2}-p_{1}\right)} \times \\
& \quad\left\{\int_{-\pi}^{\pi}|\psi(t+h)-\psi(t-h)|^{p_{2}} d t\right\}^{\left(2-p_{1}\right) /\left(p_{2}-p_{1}\right)},
\end{aligned}
$$

which is equivalent to the condition (1.7) with $p=2, q=0, k=k^{\prime}$ in Theorem 2. Hence the result follows at once.

\section{REFERENCES}

(1) K. K. Chen, On the absolute Cesàro summability of negative order for a Fourier series at a given point. Amer.J. Math. 66 (1944), 299-312. 1957).

(2) K. K. CHEN, Summation of the Fourier Series of Orthogonal Functions (Peking, $\dagger$ Since $|1-z|=\sqrt{ }\left\{(1-r)^{2}+4 \sin ^{2}(t / 2)\right\} \geqq \sqrt{ }\left(\frac{1}{2} \pi\right) . t$, we have $|1-z| \geqq K|t|$. 


\section{ABSOLUTE CESÀRO SUMMABILITY OF A FOURIER SERIES 319}

(3) H. C. Chow, Note on the absolute Cesàro summability of power series. Proc. London Math. Soc. (2) 43 (1937), 484-489.

(4) H. C. Chow, The absolute summability of Fourier series. J. London Math. Soc. 17 (1942), 17-23.

(5) T. M. FLETT, On an extension of absolute summability and some theorems of Littlewood and Paley. Proc. London Math. Soc. (3) 7 (1957), 113-141.

(6) T. M. FLETT, On the absolute summability of a Fourier series and its conjugate series. Proc. London Math. Soc. (3) 8 (1958), 257-311.

(7) T. M. FlETT, Some more theorems concerning the absolute summability of Fourier series and power series. Proc. London Math. Soc. (3) 8 (1958), 359-387.

(8) G. H. HARDY and J. E. LiTtLewood, A convergence criterion for Fourier series. Math. Zeitschrift, 28, (1928), 612-634.

(9) G. H. HARdY and J. E. LiTtLEWOod, Some properties of fractional integrals, I. Math. Zeitschrift, 27 (1928), 565-606.

(10) J. M. HysLop, On the absolute summability of Fourier series. Proc. London Math. Soc. (2) 43 (1937), 475-483.

(11) E. Kogbetliantz, Sur les séries absolument sommables par la méthode des moyennes arithmétique. Bull. des Sci. Math. (2) 49 (1925), 234-256.

(12) A. Zygmund, Remarque sur la convergence absolue des séries de Fourier. J. London Math. Soc. 3 (1928), 194-196.

Department of Mathematics

UNIVERSITY OF HONG KONG 